\title{
Systemic treatment and narrowband ultraviolet B differentially affect cardiovascular risk markers in psoriasis.
}

\author{
Gunnthorunn Sigurdardottir, Anna-Karin Ekman, Mona Ståhle, Cecilia Bivik and Charlotta \\ Enerbäck
}

\section{Linköping University Post Print}

\section{Tweet}

N.B.: When citing this work, cite the original article.

Original Publication:

Gunnthorunn Sigurdardottir, Anna-Karin Ekman, Mona Ståhle, Cecilia Bivik and Charlotta Enerbäck, Systemic treatment and narrowband ultraviolet B differentially affect cardiovascular risk markers in psoriasis., 2014, The Journal of American Academy of Dermatology, (70), 6, 10671075.

http://dx.doi.org/10.1016/j.jaad.2013.12.044

Copyright: Elsevier

http://www.elsevier.com/

Postprint available at: Linköping University Electronic Press

http://urn.kb.se/resolve?urn=urn:nbn:se:liu:diva-107732 


\section{Systemic treatment and narrowband UVB differentially affect cardiovascular risk markers in psoriasis}

Gunnthorunn Sigurdardottir, $\mathrm{MD}^{1 *}$, Anna-Karin Ekman, $\mathrm{PhD}^{1 *}$, Mona Ståhle, $\mathrm{MD} \mathrm{PhD}^{2}$, Cecilia Bivik, $\mathrm{PhD}^{1}$, Charlotta Enerbäck, $\mathrm{MD} \mathrm{PhD}^{1}$

${ }^{1}$ Ingrid Asp Psoriasis Research Center, Department of Clinical and Experimental Medicine, Faculty of Health Sciences, Linköping University, Linköping, Sweden, ${ }^{2}$ Unit of Dermatology and Venereology, Department of Medicine, Karolinska Institutet, Stockholm, Sweden

*These authors contributed equally to this work.

Corresponding author: Charlotta Enerbäck, Ingrid Asp Psoriasis Research Center, Department of Clinical and Experimental Medicine, Linköping University, SE-581 85 Linköping, Sweden Phone: +46 10103 7429, Fax: +46 10103 1718, E-mail: charlotta.enerback@liu.se

Word count: Abstract: 191, Capsule summary: 46, Text (excl abstract, references, figures): 2539, 46 references, 4 figures, 1 table

Funding sources: Ingrid Asp Foundation, Welander Foundation, Medical Research Council of South-east Sweden

Reprint requests: Corresponding author

Disclosure: Dr Ståhle has had speaking and/or advisory board engagements for Pfizer, Abbott, Novartis and Janssen, and received honoraria for this. She has also served as investigator for Pfizer and Janssen and received grants for this. Dr Sigurdardottir, Dr Ekman, Dr Bivik and Dr Enerbäck have no conflicts of interest to declare. 


\section{Abstract}

Background: Psoriasis is associated with a systemic inflammation and an increased frequency of the metabolic syndrome, both of which are believed to link psoriasis to an increased risk of cardiovascular disease.

Objective: The study aimed to investigate the systemic expression of markers of cardiovascular risk and determine their response to UVB therapy and treatment with the TNF- $\alpha$ inhibitor, etanercept.

Methods: Six markers of cardiovascular risk were measured systemically in psoriasis patients. The association of these markers with body mass index (BMI) and waist-hip ratio (WHR) was studied in a matched population. The marker levels were determined before and after treatment with UVB therapy or TNF- $\alpha$ inhibitor.

Results: Five of the six investigated markers were elevated in psoriasis patients. Four of these correlated to the BMI and WHR, suggesting a link to the metabolic syndrome. tPAI-1 remained elevated independently of these factors. The levels of the investigated risk markers dropped considerably after TNF- $\alpha$ inhibitor treatment but remained unaffected by UV therapy.

Limitations: A relatively limited study population. Non-randomized.

Conclusion: These findings suggest that the choice of treatment in psoriasis may influence the cardiovascular risk in patients with psoriasis and the metabolic syndrome.

Key words: psoriasis, cardiovascular risk, UVB, TNF- $\alpha$ inhibitor, sVCAM-1, sICAM-1, sEselectin, MMP9, MPO, tPAI-1 


\section{Abbreviations and acronyms}

$\begin{array}{ll}\text { BMI } & \text { body mass index } \\ \text { HDL } & \text { high-density lipoprotein } \\ \text { LDL } & \text { low-density lipoprotein } \\ \text { MMP9 } & \text { matrix metalloproteinase 9 } \\ \text { MPO } & \text { myeloperoxidase } \\ \text { NB-UVB } & \text { narrowband ultraviolet B } \\ \text { PASI } & \text { Psoriasis Area and Severity Index } \\ \text { sE-selectin } & \text { soluble E-selectin } \\ \text { sICAM-1 } & \text { soluble Intracellular Adhesion Molecule } 1 \\ \text { sVCAM-1 } & \text { soluble Vascular Cell Adhesion Molecule 1 } \\ \text { TNF- } \alpha & \text { tumor necrosis factor alpha } \\ \text { tPAI-1 } & \text { total Plasminogen Activator Inhibitor 1 } \\ \text { WHR } & \text { waist-hip ratio }\end{array}$




\section{Introduction}

Psoriasis is a chronic, immune-mediated inflammatory skin disease that affects $2-3 \%$ of the population worldwide. In addition to running an increased risk of psoriatic arthritis, patients with psoriasis run a higher risk of developing obesity, dyslipidemia, hypertension and diabetes, all components of the metabolic syndrome. ${ }^{1,2}$ Psoriasis may also confer an elevated risk of cardiovascular disease, such as myocardial infarction and stroke, independently of major risk factors for these diseases. An increase in mortality is observed in patients with severe disease and is most pronounced in young patients. ${ }^{3-8}$

Specific biomarkers aid in detecting disease, determining the activity and the severity and evaluating the response to therapy and monitoring disease progression. Cardiovascular biomarkers, many of which are also mediators of inflammation, have previously been studied in psoriasis patients. These studies have mainly focused on cardiovascular markers related to the obesity-associated systemic inflammation. The expression of the adipokine leptin and the soluble leptin receptor is higher in the serum of psoriasis patients ${ }^{9}$ and is most pronounced in severely affected individuals. Systemic psoriasis treatment decreases the levels of the adipokine resistin and increases the levels of the anti-inflammatory cardioprotective adipokine adiponectin. ${ }^{10}$

In a former study, we investigated circulating chemokines in psoriasis as a sign of an on-going systemic inflammation. Five chemokines of a Th1-, Th2- or Th17-associated phenotype were elevated in psoriasis plasma but not in healthy controls.

The expression of the chemokine CCL20 correlated strongly with disease severity. ${ }^{11}$ However, while these chemokines were highly expressed in psoriasis patients, they were not affected by narrowband UVB (NB-UVB) treatment, despite the relief of disease symptoms in the skin. In this 
study, selected risk-associated molecules implicated in the process of cardiovascular disease, soluble Vascular Cell Adhesion Molecule 1 (sVCAM-1), soluble Intracellular Adhesion Molecule 1 (sICAM-1), soluble E-selectin (sE-selectin), matrix metalloproteinase 9 (MMP9), myeloperoxidase (MPO) and total Plasminogen Activator Inhibitor 1 (tPAI-1), were analyzed. The aim of the study was two-fold: firstly, to assess the plasma levels of the selected cardiovascular risk molecules in patients with psoriasis compared with age-, gender-, body mass index- (BMI) and waist-hip ratio (WHR)-matched controls and, secondly, to investigate the effects of local (NBUVB therapy) and systemic (tumor necrosis factor alpha; TNF- $\alpha$ inhibitor) treatment. 


\section{Materials and methods}

\section{Study design}

All patients and controls included in the study were examined and the diagnosis of psoriasis was verified by a dermatologist at the departments of dermatology at Linköping University Hospital, Sahlgrenska University Hospital in Gothenburg or Karolinska University Hospital in Stockholm. Disease severity was assessed with the Psoriasis Area and Severity Index (PASI). The study was approved by the local ethics committee and every participant gave his/her written informed consent.

Six cardiovascular risk markers were analyzed in plasma from 28 psoriasis patients and 28 ageand gender-matched controls. This study group was complemented with additional patients and controls to enable the matching of 26 age-, gender- and BMI-matched and 13 age-, gender- and WHR-matched pairs. The patients had not received systemic TNF- $\alpha$ inhibitor treatment for at least four weeks prior to the study.

The BMI matching was performed within the range of $+/-1.0 \mathrm{~kg} / \mathrm{m}^{2}$ and the WHR matching within the range of $+/-0.05$, where both individuals in a matched pair either had WHR values within the normal range or could be defined as having abdominal obesity. ${ }^{12}$

Finally, the systemic expression of selected risk markers was quantified in 21 psoriasis patients before and after 12 weeks of treatment with NB-UVB and in 20 psoriasis patients before and after treatment with the TNF- $\alpha$ inhibitor etanercept (Enbrel, Pfizer). The patients had not received UV 
therapy or TNF- $\alpha$ inhibitor treatment for at least the previous four weeks. The study was nonrandomized and the evaluations of markers were performed unblinded.

\section{$\underline{\text { Blood samples }}$}

Blood was collected in CPT ${ }^{\mathrm{TM}}$ tubes (Becton Dickinson, Stockholm, Sweden) coated with sodium heparin anticoagulant, or in serum tubes with clot activator (Terumo Europe, Västra Frölunda, Sweden) for the isolation of plasma and serum respectively. The CPT tubes were centrifuged at 1,400 rcf for 25 minutes, separating the leukocytes from the plasma. The serum tubes were allowed to sit for 30 minutes before separating the serum from the clotted blood by centrifugation at 1,900 rcf for 10 minutes. Plasma and serum were frozen immediately after isolation and stored at $-80^{\circ} \mathrm{C}$ until analysis.

\section{Measurements of the cardiovascular risk markers}

The levels of the biomarkers, sVCAM-1, sICAM-1, sE-selectin, MMP9, MPO and tPAI-1 were measured in plasma or serum. The measurements were performed using the Milliplex ${ }^{\mathrm{TM}}$ Cardiovascular Disease Panel 1 MAP kit (Millipore Corporation, Billerica, MA, USA), according

to the manufacturer's instructions. The samples were analyzed on a Luminex 200 instrument (Biosource, Nivelles, Belgium) and the data were analyzed using the StarStation 3.0 software (Applied Cytometry, Sheffield, UK).

\section{$\underline{\text { Statistical analysis }}$}

Data analysis was performed in GraphPad Prism 5.0 (GraphPad Software, San Diego, CA, USA). Data were compared using the non-parametric Mann-Whitney U test or Wilcoxon matched-pair 
signed rank test. Correlations were determined by Spearman’s test. A p-value of less than 0.05 was considered significant. 


\section{$\underline{\text { Results }}$}

\section{$\underline{\text { Elevated cardiovascular markers in psoriasis patients }}$}

Measurements of the six selected cardiovascular risk markers in plasma from 28 psoriasis patients and 28 age- and gender-matched controls revealed increased levels of sICAM-1 ( $\mathrm{p}=0.0005)$, sEselectin $(\mathrm{p}=0.001)$, MMP9 $(\mathrm{p}<0.0001)$, MPO $(\mathrm{p}=0.0003)$ and tPAI-1 $(\mathrm{p}=0.0005)$ and a tendency towards an increase in sVCAM-1 ( $\mathrm{p}=0.07)$ in psoriasis patients (Fig. 1). None of these markers correlated significantly to PASI (data not shown), suggesting that they indeed represent increased systemic inflammation rather than being elevated as a result of the local inflammation in the skin.

To evaluate whether BMI and WHR influence the observed increase in cardiovascular risk markers, we correlated the levels of the investigated markers with the BMI and WHR of the study participants. This revealed an association between the BMI and sE-selectin $(r=0.68, p<0.0001)$. The correlation was also significant but moderate for tPAI-1 ( $\mathrm{r}=0.57, \mathrm{p}=0.0014)$, sICAM-1 $(\mathrm{r}=0.43, \mathrm{p}=0.02)$ and MMP9 $(\mathrm{r}=0.41, \mathrm{p}=0.03)$. A prominent correlation was seen between WHR and sE-selectin $(\mathrm{r}=0.75, \mathrm{p}<0.0001)$. There was also a correlation for tPAI-1 ( $\mathrm{r}=0.65, \mathrm{p}=0.0002)$, MMP9 ( $\mathrm{r}=0.51, \mathrm{p}=0.01)$ and sICAM-1, where the latter was more modest $(\mathrm{r}=0.39, \mathrm{p}=0.04)$.

\section{Elevation of tPAI-1 in psoriasis patients when adjusted for BMI and WHR}

Since the measured cardiovascular risk markers were at least partly influenced by BMI and WHR, their levels were next compared in psoriasis patients and BMI- or WHR-matched controls. We observed a significant elevation in tPAI-1 in psoriasis patients compared with both BMI-matched controls ( $p=0.01$ ) (Fig. 2) and WHR-matched controls ( $p=0.05$, data not shown). 
Reduced levels of cardiovascular markers after systemic TNF- $\alpha$ inhibitor treatment but not after $\underline{\text { UVB treatment }}$

In order to evaluate the effect of different treatments on the levels of the selected markers, measurements were made on the plasma of psoriasis patients before and after 12 weeks of NBUVB treatment and on the serum of psoriasis patients before and after systemic treatment with the TNF- $\alpha$ inhibitor, etanercept.

The treatment with both NB-UVB and the TNF- $\alpha$ inhibitor effectively reduced the PASI scores $(\mathrm{p}<0.0001$ for both groups, data not shown). There were no significant differences in the magnitude of the PASI decrement (data not shown).

After 12 weeks of UVB treatment, patients had sustained high levels of the cardiovascular markers (Fig. 3). Patients who had undergone therapy with the TNF- $\alpha$ inhibitor displayed a considerable reduction in all the investigated risk markers (Fig. 4). 


\section{Discussion}

The characterization of the cardiovascular biomarkers in psoriasis is an important step towards understanding the association between psoriatic and cardiovascular disease. In this study, we have investigated the systemic levels of six risk markers for cardiovascular disease in psoriasis patients.

There is accumulating evidence of an on-going systemic inflammation in psoriasis which is believed to be a pivotal link to the cardiovascular co-morbidities associated with the disease. Psoriasis is associated with obesity and pro-inflammatory cytokines and adipokines derived from the adipose tissue can be detected systemically in patients with psoriasis. Insulin resistance, which correlates with PASI, ${ }^{13}$ contributes to endothelial dysfunction, manifested by an imbalance in the release of vasodilating and vasoconstricting factors. This imbalance predisposes patients to development of atherosclerosis. ${ }^{14}$ A mechanistic link between the systemic inflammation and cardiovascular risk has been suggested by the anti-inflammatory and cardioprotective effect of statins in patients with normal lipid levels. ${ }^{15}$

Psoriasis shares many features with atherosclerosis in terms of immunoactivation, cytokine network and angiogenesis. ${ }^{16,17}$ Both conditions are characterized by infiltration of activated T cells and atherosclerosis macrophages, whose migration from the blood vessels is mediated by the interaction of adhesion molecules with selectins. The membrane-bound forms of ICAM-1 and VCAM-1 are located on the endothelial surface and facilitate the adhesion and transendothelial migration of leukocytes into the extra-vascular space to enable the interaction of immune cells and promote inflammation, resulting in the formation of plaque in both conditions. 
In this study, the inflammatory markers were selected as being indicative of cardiovascular risk (Table 1). Adhesion molecules, including ICAM-1, VCAM-1 and E-selectin, are recognized as mediators of inflammation in cardiovascular disease ${ }^{18}$ and the levels of their soluble forms correlate to various cardiovascular risk factors, such as hypercholesterolemia, hypertension and diabetes. ${ }^{19}$ sICAM-1 predicts future coronary artery disease, whereas sVCAM-1 is a marker of the severity of atherosclerosis in patients with established disease. ${ }^{20}$ In the psoriatic lesion, membranebound ICAM-1 has been detected in keratinocytes ${ }^{21}$ and ICAM-1 and VCAM-1 are expressed in T cells and Langerhans cells of the inflammatory infiltrate. ICAM-1, VCAM-1 and E-selectin have also been demonstrated in dermal vessels of psoriatic lesions. ${ }^{21,22}$ In a meta-analysis comprising 78 studies, increased levels of sICAM-1 and sE-selectin were detected in the serum of psoriasis patients. $^{23}$

MMPs degrade extracellular matrix components and are regarded as key regulatory molecules in the pathogenesis of cardiovascular disease. ${ }^{24}$ MMP9 levels are independently associated with adverse cardiovascular outcomes. ${ }^{25}$ The MMP9 protein is induced by TNF- $\alpha$ and is in psoriatic lesions produced by macrophages and neutrophils. ${ }^{26}$ In a study of psoriatic arthritis, infliximab treatment led to a reduction in the MMP9 and sE-selectin serum levels, together with a reduction in the spontaneous release of MMP9 and sE-selectin from lesional skin. ${ }^{27}$ Therapy with TNF- $\alpha$ inhibitor also leads to a reduction in MMP9 in peripheral blood mononuclear cells, plasma and in psoriatic lesions. ${ }^{28}$ Moreover, methotrexate downregulates these molecules in psoriatic skin. ${ }^{21}$ MPO is an enzyme present in leukocytes and in arteriosclerotic plaques, where it oxidizes lowdensity lipoprotein (LDL) in the artery wall. Elevated levels of MPO are linked to an increased risk of coronary disease and MPO has been proposed as a prognostic factor. ${ }^{29}$ Thrombus-related $^{2}$ cardiovascular markers have also been implicated in psoriasis. The most common abnormality of 
fibrinolysis is due to increased levels of PAI-1. PAI-1 is the main inhibitor of tissue-type plasminogen activator and is therefore a potent inhibitor of fibrinolysis. Elevated plasma levels are noted in abdominal obesity, insulin resistance, hypertriglyceridemia, thrombosis and cardiovascular disease $^{30}$ and may be a link between obesity, insulin resistance and a risk of cardiovascular events. ${ }^{31}$ Previous data on PAI-1 expression in psoriasis are limited. In a study by Gissler et al., which investigated PAI-1 expression in skin, PAI-1 was not detected in normal epidermis and was only present in a fraction of the psoriatic lesions investigated. ${ }^{32}$ Another study revealed increased levels of PAI-1 in the plasma of psoriatic patients compared with controls, followed by a reduction during treatment. ${ }^{33}$

While these markers are strongly associated with cardiovascular risk, it should be remembered that additional mediators may contribute.

The effect of TNF- $\alpha$ inhibitor treatment on the levels of these mediators is not surprising. TNF- $\alpha$ is a key pro-inflammatory cytokine in psoriasis and induces the expression of several inflammatory adipokines and adhesion molecules, such as ICAM-1, VCAM-1 and E-selectin. ${ }^{34}$ NB-UVB phototherapy is a standard treatment for psoriasis. It is often the prior intervention before systemic treatment is considered in patients with moderate to severe psoriasis. UVB is considered primarily to affect the epidermis, where a major part of the UVB radiation is absorbed. NB-UVB suppresses the interferon and Th17-signaling pathways in the skin. ${ }^{35}$ Moreover, keratinocyte apoptosis is a mechanism of action of NB-UVB in psoriatic epidermis. ${ }^{36}$

We demonstrate that, in spite of clinical improvements in psoriasis skin symptoms, patients receiving NB-UVB treatment display continuously high levels of circulating cardiovascular risk 
markers, supporting the hypothesis that the effect of the treatment is localized to the skin. This is in contrast to the patients treated with TNF- $\alpha$ inhibitor, where we detected highly significant reductions in the studied risk markers after treatment.

This study was a non-randomized study. As such, it cannot be fully excluded that other factors present in the etanercept group could influence the responsiveness to treatment. However, the reduction we observe in the etanercept group is very pronounced, and the notion of a decreased cardiovascular risk by use of TNF- $\alpha$ inhibitors is supported by findings of a decreased occurrence of myocardial infarction in psoriasis patients upon treatment. ${ }^{37}$ Systemic therapy has an effect on cardiocascular risk in both rheumatoid arthritis and psoriasis, both with systemic biological therapy and methotrexate. ${ }^{38,39}$ In psoriasis, methotrexate downregulates the expression of the membranebound isoforms of ICAM-1, VCAM-1 and E-selectin in the skin. ${ }^{21}$ Furthermore, the therapy decreases the soluble form of E-selectin in serum from patients with the autoimmune skin disease bullous pemphigoid. ${ }^{40}$ The cardiovascular risk biomarkers hs-CRP, VEGF and resistin decrease similarly in psoriasis both after biological therapy and methotrexate. ${ }^{10}$

Our results are also supported by the data from Romaní et al., who observed increased systemic levels of LDL cholesterol, non-HDL (high-density lipoprotein) cholesterol and Apo-B in psoriasis patients, levels which were unaffected by phototherapy. ${ }^{41}$ Signs of a persistent inflammatory process after UV therapy were also established by Coimbra et al. ${ }^{42}$ In contrast, UV therapy led to a decrease in IL-22, IL-17, IL-23, IL-8 TNF- $\alpha$, and VEGF serum levels, ${ }^{43}$ suggesting that the skin is the major source of these cytokines in psoriasis. However, the literature is controversial and both we and others have shown a lack of effect by UV therapy on the studied cytokines. ${ }^{35,44,45}$ It should be remembered that the relationship between local inflammation and systemic inflammation is complex, and that an influence from the skin 
inflammation may affect the systemic cardiovascular risk. This is demonstrated by hs-CRP, which is a systemic marker of cardiovascular risk but which strongly correlates to disease severity measured by PASI and is reduced along with skin symptoms, for example upon UVB treatment. $^{46}$

In conclusion, we observe an increase in the plasma levels of several markers associated with cardiovascular risk. Several of these markers correlate with BMI and WHR and are therefore most likely the result of the increased prevalence of the metabolic syndrome in patients with psoriasis. The levels of the markers were effectively diminished by treatment with the TNF- $\alpha$ inhibitor but they were not reduced by UVB treatment. This lack of reduction in cardiovascular risk markers after UV therapy suggests that this commonly used treatment might have a limited effect on the systemic inflammation and risk of cardiovascular co-morbidities in psoriasis. It also suggests that, in addition to alleviating psoriasis symptoms, systemic treatment with TNF- $\alpha$ inhibitors might also serve to reduce the risk of cardiovascular co-morbidities seen in psoriasis patients. Patients with psoriasis and the metabolic syndrome may therefore benefit from systemic treatment rather than UV therapy. 


\section{Acknowledgements}

This research was funded by the Ingrid Asp Foundation, the Welander Foundation and the Medical Research Council of Southeast Sweden. 


\section{Figures}

Fig. 1. The levels of cardiovascular risk markers sVCAM-1, sICAM-1, sE-selectin, MMP9, MPO and tPAI-1 measured in the plasma of age- and gender-matched psoriasis patients (Psoriasis) and controls (Controls). The line shows the median, $n=28$. The mean age of the patients was 48.1 years (range 16-78 years) and the median PASI score 7.55 (range $2.0-25.3$ ), ${ }^{* *} \mathrm{p}<0.01,{ }^{* * *} \mathrm{p}<0.001$, $* * * * \mathrm{p}<0.0001$.
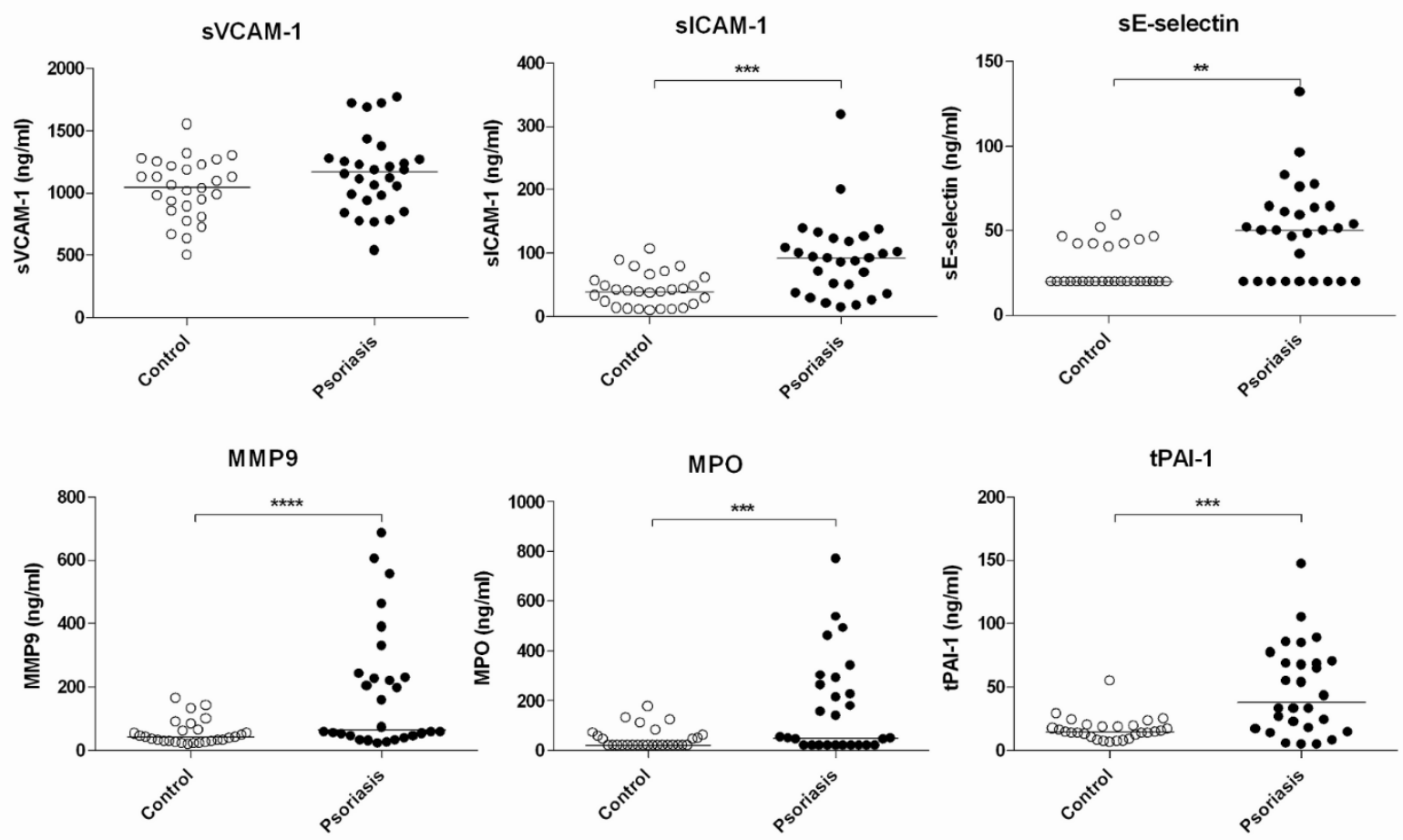
Fig. 2. The levels of cardiovascular risk markers sVCAM-1, sICAM-1, sE-selectin, MMP9, MPO and tPAI-1 measured in the plasma of age-, gender- and BMI-matched psoriasis patients (Psoriasis) and controls (Control), $\mathrm{n}=26$. The line shows the median. The mean age and BMI of the patients were 50.7 years (range $27-79$ ) and $26.0 \mathrm{~kg} / \mathrm{m}^{2}$ (range 22.0-34.7). The median PASI score was 6.7 (range $0.6-17.1),{ }^{*} \mathrm{p}<0.05$.
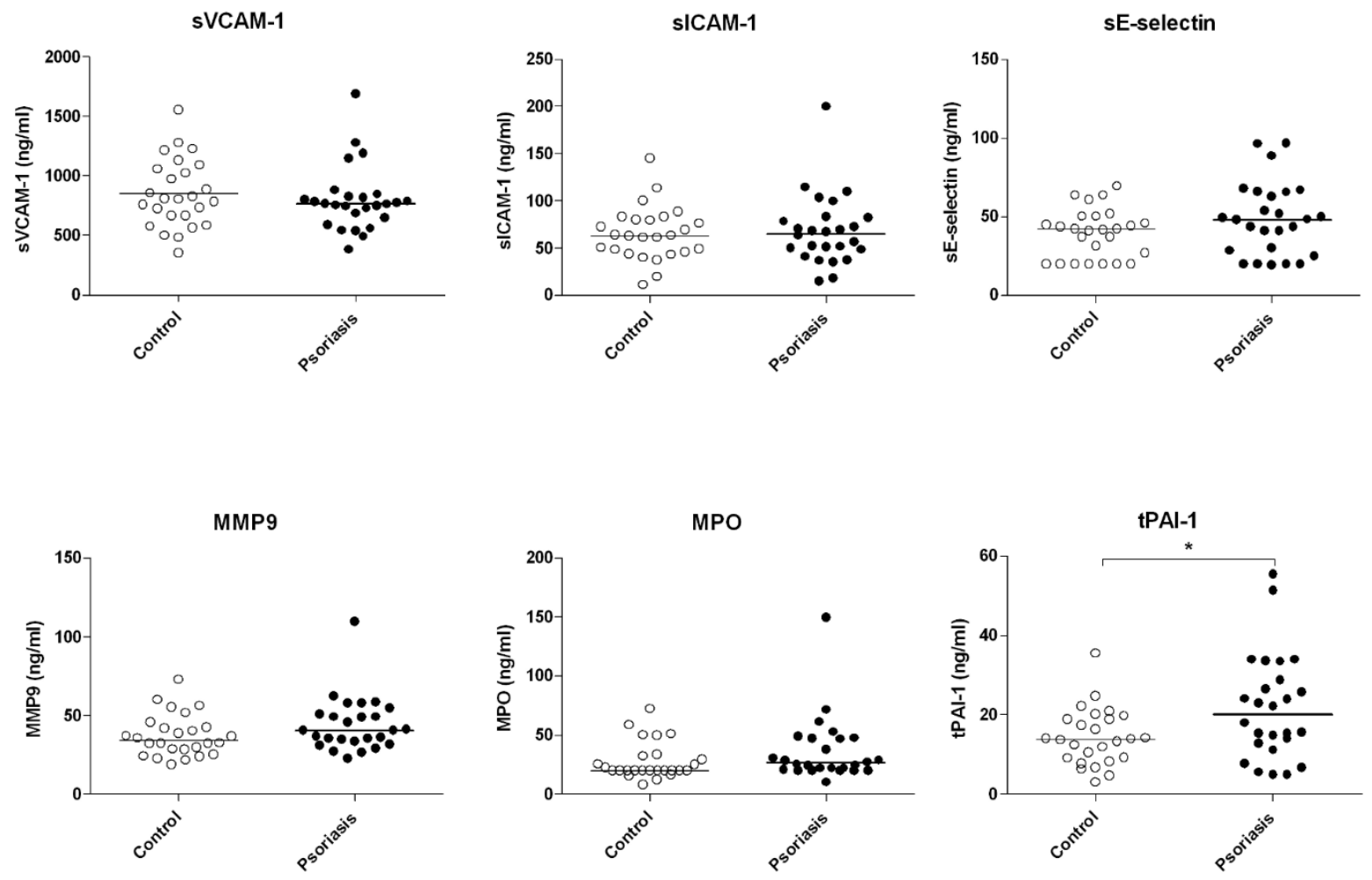
Fig. 3. The levels of cardiovascular risk markers sVCAM-1, sICAM-1, sE-selectin, MMP9, MPO and tPAI-1 in the plasma of psoriasis patients before (Before UVB) and 12 weeks after NB-UVB therapy (After UVB, 12 weeks), $n=21$. The mean age of the patients was 50.2 years. The median PASI was 8.3 (range 2.20-17.2) at week 0 and 1.8 (range $0.0-5.6$ ) at week 12.
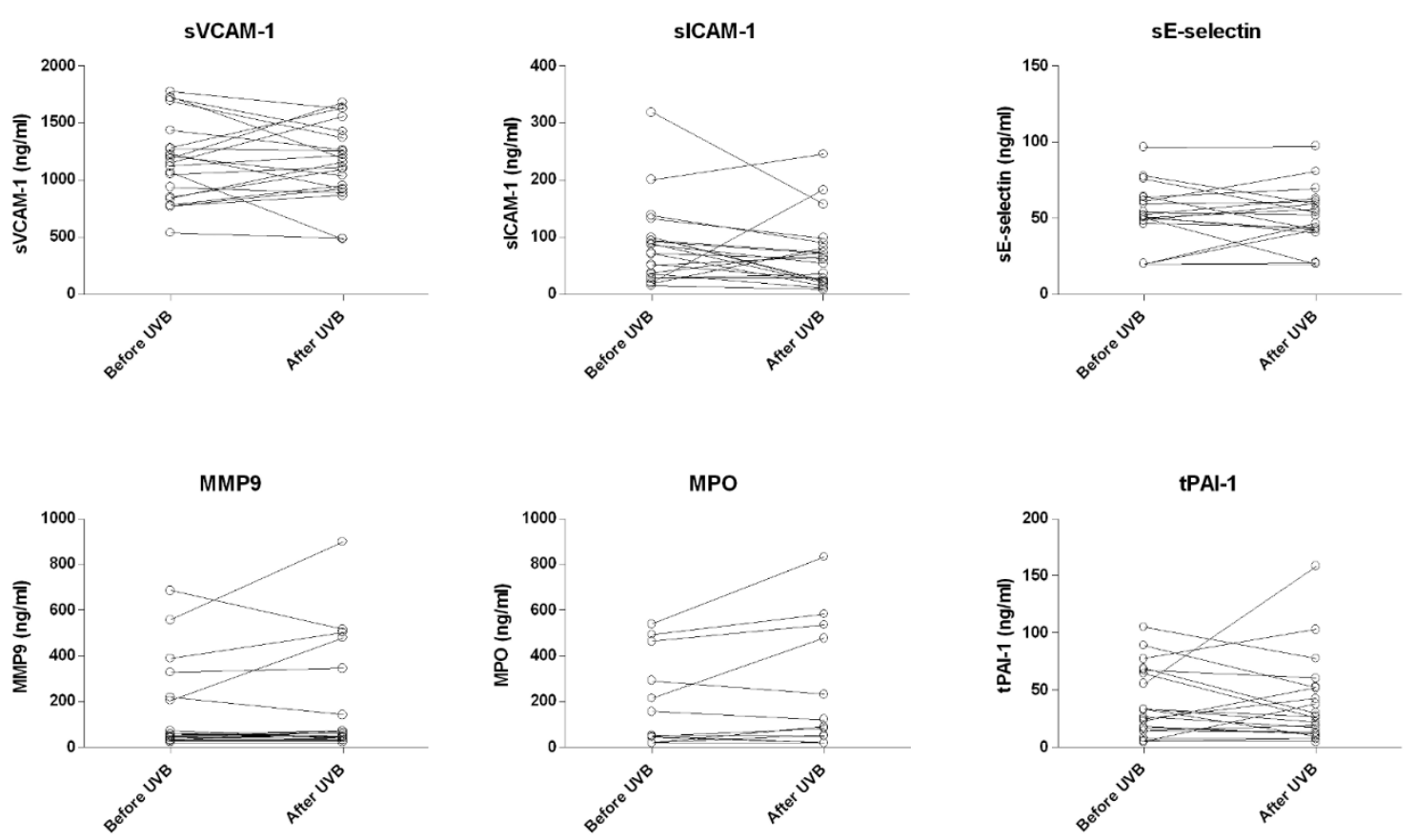
Fig. 4. The levels of cardiovascular risk markers sVCAM-1, sICAM-1, sE-selectin, MMP9, MPO and tPAI-1 in the serum of psoriasis patients before (Before Enbrel) and after treatment with etanercept (After Enbrel), n=20. The mean age of the patients was 46.4 years. The median PASI was 12.0 (range 5.0-22.4) before treatment and $4.0(0.6-11.4)$ after treatment, $* * \mathrm{p}<0.01$, $* * * \mathrm{p}<0.001, * * * * \mathrm{p}<0.0001$
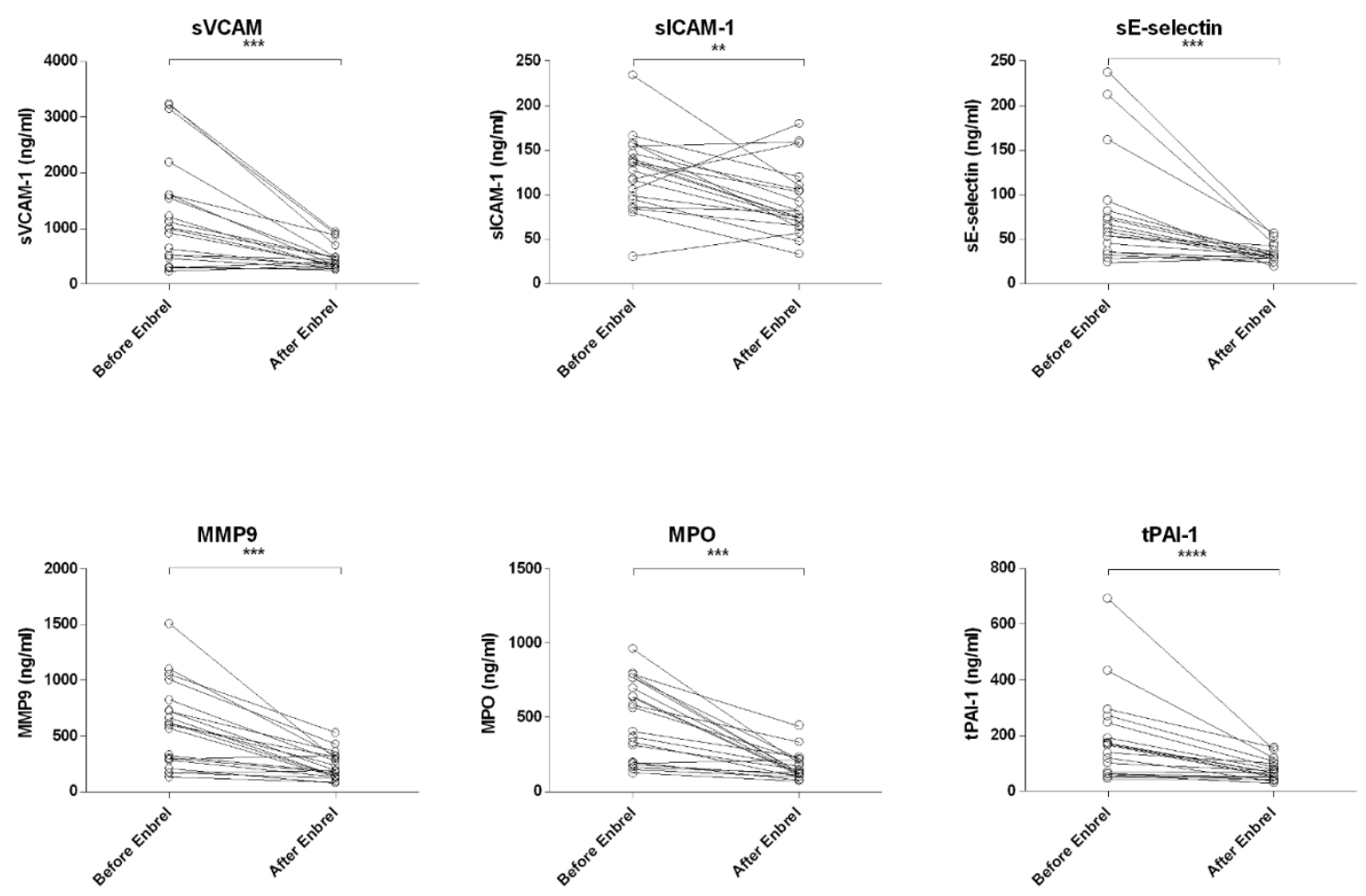
Table 1. Inflammatory markers analyzed.

\begin{tabular}{|c|c|c|}
\hline Inflammatory marker & Indicated cardiovascular risk & References \\
\hline $\begin{array}{l}\text { vascular cell adhesion molecule } 1 \\
\text { (VCAM-1) }\end{array}$ & \multirow{3}{*}{$\begin{array}{l}\text {-inflammatory mediators in cardiovascular } \\
\text { disease } \\
\text {-the levels correlate to various cardiovascular } \\
\text { risk factors, such as hypercholesterolemia, } \\
\text { hypertension and diabetes }\end{array}$} & \multirow[t]{3}{*}{17} \\
\hline $\begin{array}{l}\text { intracellular adhesion molecule } 1 \\
\text { (ICAM-1) }\end{array}$ & & \\
\hline E-selectin & & \\
\hline $\begin{array}{l}\text { matrix metalloproteinase } 9 \\
\text { (MMP9) }\end{array}$ & $\begin{array}{l}\text {-associated with increased risk for stroke or } \\
\text { cardiovascular death }\end{array}$ & 24 \\
\hline myeloperoxidase (MPO) & $\begin{array}{l}\text {-present in arteriosclerotic plaques } \\
\text {-associated with increased risk of coronary } \\
\text { disease }\end{array}$ & $\begin{array}{l}28 \\
28\end{array}$ \\
\hline $\begin{array}{l}\text { total plasminogen activator } \\
\text { inhibitor } 1 \text { (tPAl-1) }\end{array}$ & $\begin{array}{l}\text {-elevated levels in thrombosis and } \\
\text { cardiovascular disease } \\
\text {-increased levels are suggested as a link } \\
\text { between obesity, insulin resistance and a risk } \\
\text { of cardiovascular events } \\
\text {-elevated levels in atherosclerotic vessel walls } \\
\text { and atherosclerotic lesions }\end{array}$ & $\begin{array}{l}29 \\
30\end{array}$ \\
\hline
\end{tabular}




\section{References}

1. Menter A, Griffiths CE, Tebbey PW, Horn EJ, Sterry W, and International Psoriasis C. Exploring the association between cardiovascular and other disease-related risk factors in the psoriasis population: the need for increased understanding across the medical community. J Eur Acad Dermatol Venereol 2010;24(12):1371-7.

2. Neimann AL, Shin DB, Wang X, Margolis DJ, Troxel AB, and Gelfand JM. Prevalence of cardiovascular risk factors in patients with psoriasis. J Am Acad Dermatol 2006;55(5):82935.

3. Mallbris L, Akre O, Granath F, Yin L, Lindelof B, Ekbom A, et al. Increased risk for cardiovascular mortality in psoriasis inpatients but not in outpatients. Eur J Epidemiol 2004;19(3):225-30.

4. Gelfand JM, Dommasch ED, Shin DB, Azfar RS, Kurd SK, Wang X, et al. The risk of stroke in patients with psoriasis. J Invest Dermatol 2009;129(10):2411-8.

5. Gelfand JM, Neimann AL, Shin DB, Wang X, Margolis DJ, and Troxel AB. Risk of myocardial infarction in patients with psoriasis. JAMA 2006;296(14):1735-41.

6. Gelfand JM, Troxel AB, Lewis JD, Kurd SK, Shin DB, Wang X, et al. The risk of mortality in patients with psoriasis: results from a population-based study. Arch Dermatol 2007;143(12):1493-9.

7. Mehta NN, Azfar RS, Shin DB, Neimann AL, Troxel AB, and Gelfand JM. Patients with severe psoriasis are at increased risk of cardiovascular mortality: cohort study using the General Practice Research Database. Eur Heart J 2010;31(8):1000-6.

8. Prodanovich S, Kirsner RS, Kravetz JD, Ma F, Martinez L, and Federman DG. Association of psoriasis with coronary artery, cerebrovascular, and peripheral vascular diseases and mortality. Arch Dermatol 2009;145(6):700-3.

9. Wang Y, Chen J, Zhao Y, Geng L, Song F, and Chen HD. Psoriasis is associated with increased levels of serum leptin. Br J Dermatol 2008;158(5):1134-5.

10. Boehncke S, Salgo R, Garbaraviciene J, Beschmann H, Hardt K, Diehl S, et al. Effective continuous systemic therapy of severe plaque-type psoriasis is accompanied by amelioration of biomarkers of cardiovascular risk: results of a prospective longitudinal observational study. J Eur Acad Dermatol Venereol 2011;25(10):1187-93.

11. Ekman AK, Sigurdardottir G, Carlstrom M, Kartul N, Jenmalm MC, and Enerback C. Systemically Elevated Th1-, Th2- and Th17-associated Chemokines in Psoriasis Vulgaris Before and After Ultraviolet B Treatment. Acta Derm Venereol 2013.

12. World Health Organization. Waist Circumference and Waist-hip Ratio: Report of a WHO Expert Consultation, Geneva, 8-11 December 2008. 2011.

13. Boehncke S, Thaci D, Beschmann H, Ludwig RJ, Ackermann H, Badenhoop K, et al. Psoriasis patients show signs of insulin resistance. Br J Dermatol 2007;157(6):1249-51.

14. Boehncke WH, Boehncke S, Tobin AM, and Kirby B. The 'psoriatic march': a concept of how severe psoriasis may drive cardiovascular comorbidity. Exp Dermatol 2011;20(4):303-7.

15. Albert MA, Danielson E, Rifai N, Ridker PM, and Investigators P. Effect of statin therapy on C-reactive protein levels: the pravastatin inflammation/CRP evaluation (PRINCE): a randomized trial and cohort study. JAMA 2001;286(1):64-70.

16. Ghazizadeh R, Shimizu H, Tosa M, and Ghazizadeh M. Pathogenic mechanisms shared between psoriasis and cardiovascular disease. Int J Med Sci 2010;7(5):284-9. 
17. Späh F. Inflammation in atherosclerosis and psoriasis: common pathogenic mechanisms and the potential for an integrated treatment approach. Br J Dermatol 2008;159 Suppl 2:107.

18. Malik I, Danesh J, Whincup P, Bhatia V, Papacosta O, Walker M, et al. Soluble adhesion molecules and prediction of coronary heart disease: a prospective study and meta-analysis. Lancet 2001;358(9286):971-6.

19. Hope SA and Meredith IT. Cellular adhesion molecules and cardiovascular disease. Part II. Their association with conventional and emerging risk factors, acute coronary events and cardiovascular risk prediction. Intern Med J 2003;33(9-10):450-62.

20. Blankenberg S, Rupprecht HJ, Bickel C, Peetz D, Hafner G, Tiret L, et al. Circulating cell adhesion molecules and death in patients with coronary artery disease. Circulation 2001;104(12):1336-42.

21. Torres-Alvarez B, Castanedo-Cazares JP, Fuentes-Ahumada C, and Moncada B. The effect of methotrexate on the expression of cell adhesion molecules and activation molecule CD69 in psoriasis. J Eur Acad Dermatol Venereol 2007;21(3):334-9.

22. de Boer OJ, Wakelkamp IM, Pals ST, Claessen N, Bos JD, and Das PK. Increased expression of adhesion receptors in both lesional and non-lesional psoriatic skin. Arch Dermatol Res 1994;286(6):304-11.

23. Dowlatshahi EA, van der Voort EA, Arends LR, and Nijsten T. Markers of systemic inflammation in psoriasis: a systematic review and meta-analysis. $\mathrm{Br} \mathrm{J}$ Dermatol 2013;169(2):266-82.

24. Tayebjee MH, Lip GY, and MacFadyen RJ. Matrix metalloproteinases in coronary artery disease: clinical and therapeutic implications and pathological significance. Curr Med Chem 2005;12(8):917-25.

25. Eldrup N, Gronholdt ML, Sillesen H, and Nordestgaard BG. Elevated matrix metalloproteinase-9 associated with stroke or cardiovascular death in patients with carotid stenosis. Circulation 2006;114(17):1847-54.

26. Suomela S, Kariniemi AL, Snellman E, and Saarialho-Kere U. Metalloelastase (MMP-12) and 92-kDa gelatinase (MMP-9) as well as their inhibitors, TIMP-1 and -3, are expressed in psoriatic lesions. Exp Dermatol 2001;10(3):175-83.

27. Cordiali-Fei P, Trento E, D'Agosto G, Bordignon V, Mussi A, Ardigo M, et al. Effective therapy with anti-TNF-alpha in patients with psoriatic arthritis is associated with decreased levels of metalloproteinases and angiogenic cytokines in the sera and skin lesions. Ann $\mathrm{N}$ Y Acad Sci 2007;1110:578-89.

28. Buommino E, De Filippis A, Gaudiello F, Balato A, Balato N, Tufano MA, et al. Modification of osteopontin and MMP-9 levels in patients with psoriasis on anti-TNFalpha therapy. Arch Dermatol Res 2012;304(6):481-5.

29. Baldus S, Heeschen C, Meinertz T, Zeiher AM, Eiserich JP, Munzel T, et al. Myeloperoxidase serum levels predict risk in patients with acute coronary syndromes. Circulation 2003;108(12):1440-5.

30. Declerck PJ and Gils A. Three decades of research on plasminogen activator inhibitor-1: a multifaceted serpin. Semin Thromb Hemost 2013;39(4):356-64.

31. Alessi MC, Peiretti F, Morange P, Henry M, Nalbone G, and Juhan-Vague I. Production of plasminogen activator inhibitor 1 by human adipose tissue: possible link between visceral fat accumulation and vascular disease. Diabetes 1997;46(5):860-7. 
32. Gissler HM, Frank R, and Kramer MD. Immunohistochemical characterization of the plasminogen activator system in psoriatic epidermis. Br J Dermatol 1993;128(6):612-8.

33. Nielsen HJ, Christensen IJ, Svendsen MN, Hansen U, Werther K, Brunner N, et al. Elevated plasma levels of vascular endothelial growth factor and plasminogen activator inhibitor-1 decrease during improvement of psoriasis. Inflamm Res 2002;51(11):563-7.

34. Gerdes S, Rostami-Yazdi M, and Mrowietz U. Adipokines and psoriasis. Exp Dermatol 2011;20(2):81-7.

35. Racz E, Prens EP, Kurek D, Kant M, de Ridder D, Mourits S, et al. Effective treatment of psoriasis with narrow-band UVB phototherapy is linked to suppression of the IFN and Th17 pathways. J Invest Dermatol 2011;131(7):1547-58.

36. Weatherhead SC, Farr PM, Jamieson D, Hallinan JS, Lloyd JJ, Wipat A, et al. Keratinocyte apoptosis in epidermal remodeling and clearance of psoriasis induced by UV radiation. $\mathrm{J}$ Invest Dermatol 2011;131(9):1916-26.

37. Wu JJ, Poon KYT, Channual JC, and Shen AYJ. Association Between Tumor Necrosis Factor Inhibitor Therapy and Myocardial Infarction Risk in Patients With Psoriasis. Archives of Dermatology 2012;148(11):1244-1250.

38. Jacobsson LT, Turesson C, Nilsson JA, Petersson IF, Lindqvist E, Saxne T, et al. Treatment with TNF blockers and mortality risk in patients with rheumatoid arthritis. Ann Rheum Dis 2007;66(5):670-5.

39. Prodanovich S, Ma F, Taylor JR, Pezon C, Fasihi T, and Kirsner RS. Methotrexate reduces incidence of vascular diseases in veterans with psoriasis or rheumatoid arthritis. J Am Acad Dermatol 2005;52(2):262-7.

40. Dahlman-Ghozlan K, Heilborn JD, and Stephansson E. Circulating levels of soluble Eselectin, ICAM-1 and VCAM-1 in bullous pemphigoid during low-dose methotrexate therapy. A prospective study. Exp Dermatol 2000;9(5):336-40.

41. Romani J, Caixas A, Carrascosa JM, Ribera M, Rigla M, and Luelmo J. Effect of narrowband ultraviolet $\mathrm{B}$ therapy on inflammatory markers and body fat composition in moderate to severe psoriasis. Br J Dermatol 2012;166(6):1237-44.

42. Coimbra S, Oliveira H, Reis F, Belo L, Rocha S, Quintanilha A, et al. Psoriasis therapy and cardiovascular risk factors: a 12-week follow-up study. Am J Clin Dermatol 2010;11(6):423-32.

43. Coimbra S, Oliveira H, Reis F, Belo L, Rocha S, Quintanilha A, et al. Interleukin (IL)-22, IL-17, IL-23, IL-8, vascular endothelial growth factor and tumour necrosis factor-alpha levels in patients with psoriasis before, during and after psoralen-ultraviolet $\mathrm{A}$ and narrowband ultraviolet B therapy. Br J Dermatol 2010;163(6):1282-90.

44. Anderson KS, Petersson S, Wong J, Shubbar E, Lokko NN, Carlstrom M, et al. Elevation of serum epidermal growth factor and interleukin 1 receptor antagonist in active psoriasis vulgaris. Br J Dermatol 2010;163(5):1085-9.

45. Enerback C. Soluble biomarkers in psoriasis. Eur J Dermatol 2011;21(6):844-50.

46. Coimbra S, Oliveira H, Belo L, Figueiredo A, Rocha-Pereira P, and Santos-Silva A. Principal determinants of the length of remission of psoriasis vulgaris after topical, NBUVB, and PUVA therapy: a follow-up study. Am J Clin Dermatol 2013;14(1):49-53. 